\title{
Refresher
}

\section{Trauma mit Folgen: das komplexe regionale}

Violeta Dimova und Frank Birklein

\author{
In einigen Fällen tritt nach einem Trauma einer Extremität das komplexe regionale Schmerzsyndrom \\ auf - Eine erfolgreiche Therapie motiviert die Patienten zum Selbstmanagement
}

Das komplexe regionale Schmerzsyndrom (CRPS) ist eine seltene Schmerzerkrankung einer Extremität. Sie entsteht nach einer Verletzung und ist in ihrer akuten Form durch entzündliche Symptome gekennzeichnet. Die Inzidenz liegt zwischen 5,5 [1] und 26,22 [2] pro 100000 Fälle im Jahr. Etwa $2 \%$ aller Menschen entwickeln nach einem Trauma ein CRPS. Bei $11 \%$ davon ist eine distale Radiusfraktur die damit häufigste Ursache, bei Nervenläsion in bis zu 5\% der Fälle [2]. Erwachsene Patienten mit komplizierten Frakturen, einer rheumatologischen Vorerkrankung oder starken Akutschmerzen ( $>5$ auf einer 11-Punkte-NRS) bis zu einer Woche nach dem Trauma haben ein erhöhtes Risiko, ein CRPS zu entwickeln [3].

Die Prognose ist im Ganzen positiv. 70\% der Betroffenen erfahren in den ersten 12 Monaten eine signifikante Verbesserung des Schmerzes, der entzündlichen Symptome und der Funktion der Extremität. Allerdings sind nur $5 \%$ innerhalb von einem Jahr komplett beschwerdefrei und nur wenige erholen sich vollständig [4].

\section{Klinische Manifestation}

Das Leitsymptom des CRPS ist der posttraumatische Schmerz. Die Patienten beschreiben ihn meistens als dauerhaft mit Intensitätsveränderungen. In der Akutphase wird er oft als tiefer Gewebeschmerz empfunden. Er verstärkt sich bei Bewegung, Temperaturveränderung (Wärme oder Kälte) sowie bei physiologischer oder psychologischer Belastung. Markante Symptome der entzündlichen Reaktion sind Ödem, Überwärmung und Schwitzen der betroffenen Extremität (Abb. 2). Sichtbar sind weiterhin trophische Veränderungen der Nägel, Behaarung und Haut (zum Beispiel Geschwüre in chronischen Stadien). Vorwiegend als Akutsymptom zeigt sich eine Hautfarbenveränderung, entweder rötlich oder blau-livide. Passend dazu ist die Hauttemperatur entweder wär-

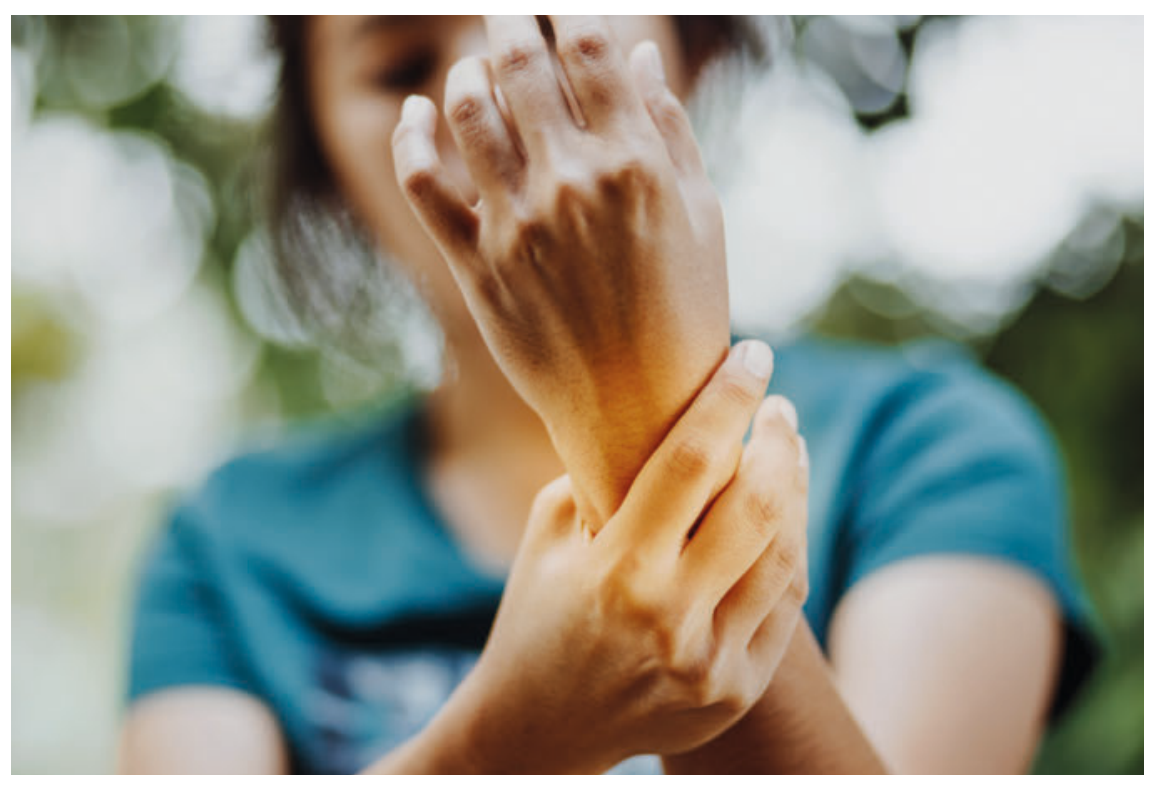

Abb. 1 Anut21ngphoto/Adobe Stock; Symbolbild

mer oder kälter verglichen mit der Gegenseite.

Das Ödem wird von Patienten mit chronischem CRPS stärker empfunden als es objektiv ist. Oft haben sich dann aber eine Allodynie und ein Funktionsverlust der Extremität entwickelt. Ausnahmslos bei allen Patienten liegen Kraftminderung und Minderbewegung vor, was die Entwicklung von Gelenkkontrakturen zur Folge hat. Seltener sind motorische Symptome wie Tremor, Myoklonien oder Dystonien [5]. Trotz Schmerz und Hyperalgesie sind Hypästhesie und Thermhypästhesie nachweisbar [6], die einem Handschuh- oder Socken-förmigen Muster folgen. Die Patienten empfinden ihre betroffene Extremität nicht mehr als dem eigenen Körper zugehörig. Abb. 2 zeigt die Kernsymptome eines CRPS.

\section{Diagnosestellung}

Die Diagnose CRPS wird derzeit klinisch anhand der von der International Association for the Study of Pain (IASP) anerkannten „Bu-
dapest-Kriterien“ [7] gestellt (s. Kasten). Ohne das Vorliegen einer mindestens bewegungsabhängigen Schmerzsymptomatik kann CRPS nicht diagnostiziert werden. Ein CRPS kann in Typ I ohne und Typ II mit nachweisbarer Nervenläsion klassifiziert werden. Zusätzlich kann zwischen einem „primär warmem" oder „primär kaltem“ Subtyp unterschieden werden, wenn Patienten retrospektiv entweder über eine initial erhöhte oder erniedrigte Hauttemperatur der Extremität berichten. Der „primär kalte“ Subtyp ist seltener. Eine sorgfältige differenzialdiagnostische Klärung ist äußerst relevant, insbesondere bei spontan auftretenden CRPS-Erkrankungen, die sehr selten vorkommen. Bei diagnostischer Unsicherheit sind folgende apparative Untersuchungen besonders hilfreich:

- 3-Phasen-Knochenszintigrafie:

Der typische Befund ist ein erhöhter Knochenstoffwechsel in der späten Mineralisationsphase in den distalen Gelenken. 


\section{Schmerzsyndrom}

\section{Zusammenfassung}

Das komplexe regionale Schmerzsyndrom (CRPS) ist eine seltene Schmerzerkrankung einer Extremität, die nach einer Verletzung entsteht. Etwa $2 \%$ aller Menschen entwickeln nach einem Trauma ein CRPS. Die Prognose der Erkrankung ist positiv - auch wenn sich die meisten Patienten nicht vollständig erholen, so ist doch eine wesentliche Besserung der Symptome bei frühzeitiger adäquater Behandlung zu erreichen.

Der Refresher gibt ein Update zu Pathophysiologie, Diagnostik, klinischer Manifestation, Chronifizierungsfaktoren und Therapie.

- MRT-Untersuchung der Extremitäten: Ausschluss rheumatischer Erkrankungen oder Infektionen.

\section{Pathophysiologie}

Eine eindeutige genetische Disposition ist bis dato nicht nachgewiesen. Die posttraumatische Entzündung ist der pathophysiologische Mechanismus des akuten CRPS. Durch die vom Trauma induzierte Immunreaktion werden Entzündungsmediatoren freigesetzt, die eine Proliferation von Bindegewebszellen auslösen und zu den typischen Kontrakturen führen, periphere Nozizeptoren sensibilisieren und Schmerz sowie Hyperalgesie auslösen. Sie erhöhen außerdem die Freisetzung von Neuropeptiden wie Calcitonin Gene-Related Peptide (CGRP) und Substanz P (SP), was Rötung, Überwärmung, Ödeme, Haarwachstum und Schwitzen verursacht [8].

Zeitgleich wird die Extremität geschont. Das verschafft zwar kurzfristig eine Symptomlinderung, langfristig fördert es jedoch Funktionsstörungen. Parallel beginnen maladaptive zentralnervöse plastische Prozesse, die das klinische Bild im weiteren Verlauf prägen:

- Bewegungsstörungen entstehen durch erlernte schmerzassoziierte Vermeidung.

- Flexionsdystonien entstehen durch eine unphysiologische Kraft-Rückkopplungsregulation ausgehend von Golgi-Sehnenorganen via spinale Reflexbögen.
- Körperrepräsentationsstörungen wie Entfremdungsgefühle der Extremität oder Überschätzung von deren Größe manifestieren sich [9].

- Eine Allodynie wird ein häufiges Symptom.

\section{Chronifizierungsfaktoren}

Psychologische Faktoren und maladaptives Lernen stehen im Herzen der CRPS-Chronifizierung. Die Schmerzangst beschreibt eine ängstliche Einstellung einer Person gegenüber Schmerzen, die sich zum Beispiel in der Befürchtung schlimmer Konsequenzen durch den Schmerz äußert. Die Schmerzangst beeinflusst den CRPS-Krankheitsverlauf nachweislich negativ [10]. Je stärker die Angst vor Schmerzen bei einem CRPS-Patienten ist, desto schneller manifestiert sich ein schmerzassoziiertes Vermeidungsverhalten.

\section{Budapest-Kriterien}

Die klinischen „Budapest-Kriterien“ zur Diagnosestellung eines CRPS nach Harden et al. [7] weisen eine Sensitivität von $99 \%$ und eine Spezifität von $68 \%$ zur Differenzierung gegen neuropathische Schmerzerkrankungen auf.

\section{Kriterium I: Anhaltender Schmerz}

- disproportional stark zum erwarteten Heilungsverlauf ausgeprägt und durch das Anfangstrauma nicht mehr erklärbar

Kriterium II: Anamnestisch berichtet der Patient über jeweils mindestens 1 Symptom aus $\mathbf{3}$ der $\mathbf{4}$ folgenden Kategorien:

1. Hyperalgesie (Überempfindlichkeit für Schmerzreize); Hyperästhesie/Allodynie (Überempfindlichkeit für Berührung)

2. Asymmetrie der Hauttemperatur; Veränderung der Hautfarbe

3. Asymmetrie des lokalen Schwitzens; Ödem
Die Bewegung der betroffenen Extremität wird reduziert oder komplett ausgelassen, um die bewegungsabhängige Verstärkung der Schmerzen zu vermeiden.

Im Verlauf entsteht oder verstärkt sich eine depressive Verstimmung. Eine klinisch relevante psychologische Traumatisierung in der Vorgeschichte berichten $38 \%$ der CRPS-Patienten, nur 10\% der Patienten mit anderen Extremitätenschmerzen und 4\% der Gesunden [11]. Es ist wahrscheinlich, dass dies die psychologische Bewältigung der CRPS-Schmerzerkrankung erschwert.

\section{Therapie}

Die CRPS-Behandlung ist idealerweise multimodal. Interventionelle Behandlungsformen können dabei als Add-on-Therapeutika in besonderen Fällen, aber grundsätzlich nicht in der Routinetherapie eingesetzt wer-
4. Reduzierte Beweglichkeit, Dystonie, Tremor, „Paresen“ (i. S. von Schwäche); Veränderungen von Haar- oder Nagelwachstum

Kriterium III: Zum Zeitpunkt der Untersuchung liegt beim Patienten jeweils mindestens 1 Symptom aus $\mathbf{2}$ der $\mathbf{4}$ folgenden Kategorien vor:

1. Hyperalgesie auf spitze Reize (z. B. Zahnstocher); Allodynie; Schmerz bei Druck auf Gelenke/Knochen/Muskeln

2. Asymmetrie der Hauttemperatur durch Palpation; Veränderung der Hautfarbe

3. Asymmetrie des lokalen Schwitzens; Ödem

4. Reduzierte Beweglichkeit, Dystonie, Tremor, „Paresen“ (i. S. von Schwäche); Veränderungen von Haar- oder Nagelwachstum

Kriterium IV: Eine andere Erkrankung erklärt die Symptomatik nicht hinreichend. 


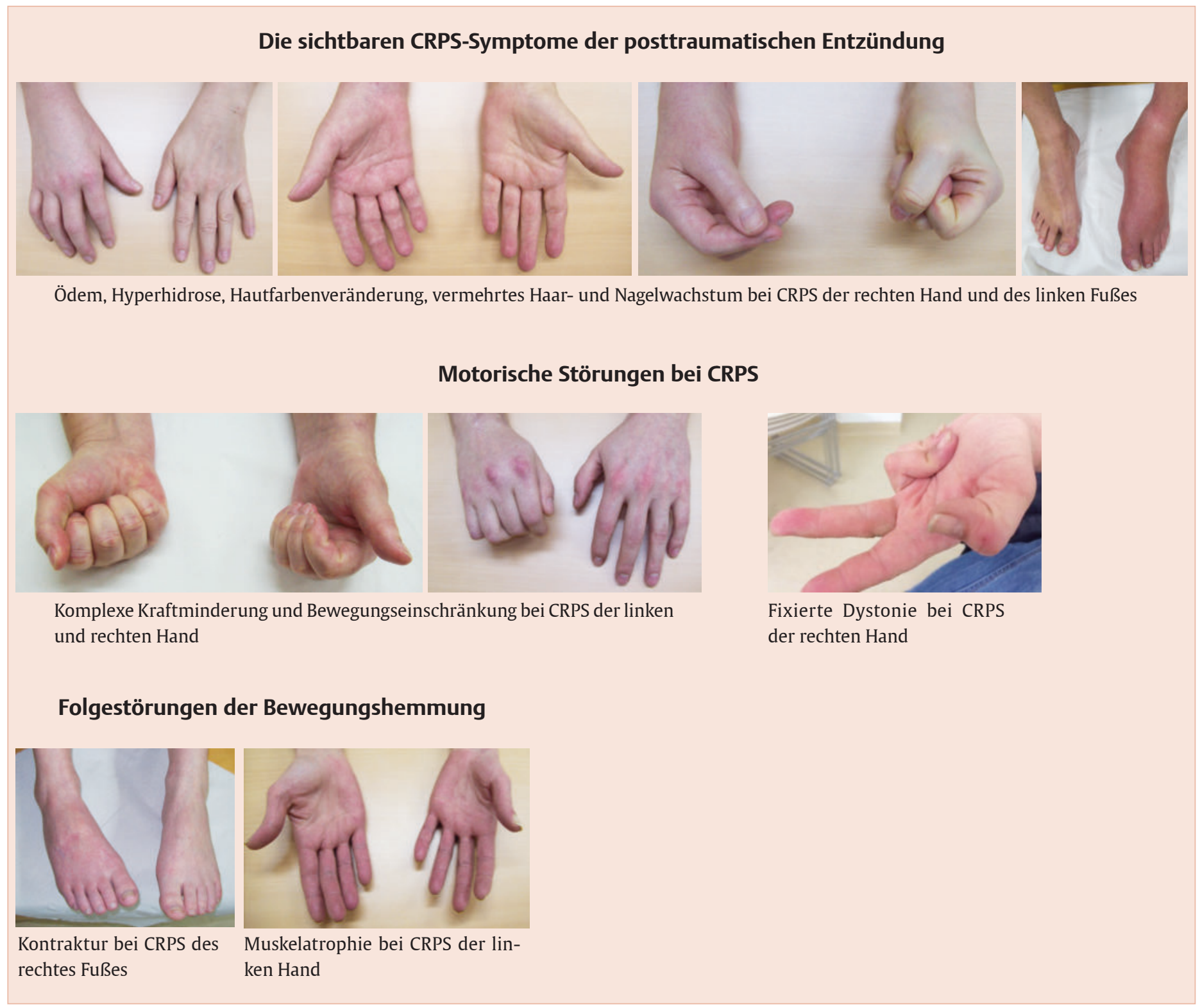

Abb. 2 Die Kernsymptome eines CRPS. @ Dimova/Birklein

\section{Der Behandlungserfolg hängt wesentlich von der Motivation und der selbst- verantwortlichen aktiven Mitbeteiligung des Patienten zur Funktionsverbes- serung der betroffenen Extremität ab.}

den. Die wichtigsten Therapieziele für die Patienten sind es, Schmerzen zu reduzieren und Funktionen wiederzuerlangen. Vom Beginn der Behandlung an gilt das Prinzip vom „Management zum Selbstmanagement“: Die Patienten sollten motiviert werden, passive Rollen zu verlassen und sich aktiv an der Krankheitsbehandlung zu beteiligen. Sie werden aufgefordert, die betroffene Extremität zu benutzen, auch wenn dies zu einer vo- rübergehenden Zunahme der Schmerzen und anderer Symptome führt.

Basierend auf der S1-Leitlinie der Deutschen Gesellschaft für Neurologie zur Diagnose und Therapie des CRPS [12] schlagen wir folgenden Therapiealgorithmus vor:

\section{Standardtherapie}

Medikamentöse und nicht-medikamentöse Schmerztherapie (akute und chronische Pha- se), welche allerdings nicht speziell für CRPS untersucht wurde:

- Gabapentin (1200-2400 (3600) mg/Tag) hat einen Effekt vor allem bei Allodynie [13]. Trizyklische Antidepressiva sind bei begleitenden Schlafstörungen hilfreich.

- Bei Opioidgabe muss eine Schmerzreduktion $>50 \%$ innerhalb von 2 Wochen erreicht werden. CAVE: Opioid-insensi- 
tive Schmerzen sind bei CRPS eher die Regel als die Ausnahme.

- Eine epidurale Rückenmarkstimulation bzw. die Stimulation abhängiger Hinterwurzelganglien ist bei CRPS der unteren Extremität geeignet, wenn die nicht-invasive Schmerztherapie versagt hat. Wir verweisen auf die S3 Leitlinie zur Epiduralen Rückenmarkstimulation [14].

Physiotherapie, Ergotherapie und Trainingstherapie in der akuten und chronischen Phase:

- Die physikalische Therapie sollte mindestens 3 Tage pro Woche stattfinden, um den Patienten nicht zu überfordern, aber zum Selbstmanagement anzuleiten.

- Ziel ist die Besserung bzw. Prävention von Kontrakturen und passiven Bewegungseinschränkungen oder Bewegungsvermeidung.

- Etablierte und wirksame Therapien sind Spiegeltherapie [15], „Graded Motor Imagery (GMI)“-Therapie [16], Graded Exposure in vivo (GEXP, siehe unten).

Antientzündliche Therapie (akute Phase bis ungefähr 6 Monate):

- Die Entzündung kann mit Steroiden, Bisphosphonaten und Dimethyl-
sulfoxid-Creme (DMSO, 50\% in fetthaltiger Basiscreme, 3-mal täglich) behandelt werden. Es gelten die typischen Kontraindikationen.

- Die Autoren empfehlen in der Akutphase die Steroidtherapie. Wir stützen uns dabei auf Interventionsstudien und eigene positive Erfahrungen mit der Verabreichung mittelhoher Dosen (100 mg Prednisolon/Tag oral, Reduktion um $25 \mathrm{mg}$ alle 4 Tage).

\section{Weiterführende Therapie}

Psychotherapie, spezifisch Therapie schmerzbezogener Ängste und Bewegungsvermeidung:

- In der Regel standardisierte kognitiv-behaviorale Schmerzpsychotherapie

- Spezifisch effektiv für das Bewegungsvermeidungsverhalten ist eine multidisziplinäre Form der schrittweisen Exposition in vivo mit physiotherapeutischen Elementen, Graded Exposure Therapy (GEXP) [17].

Sympathikusblockaden sind nicht Therapie der ersten Wahl bei CRPS:

- Solche können jedoch in Einzelfällen angewandt werden: Unter strenger Kontrolle der therapeutischen Wirkung (Schmerzreduktion jeweils um 50\%) können sie 2-mal pro Woche über 5 Wochen hinweg durch erfahrene
Schmerztherapeuten und nach erfolgreicher Testblockade versucht werden.

Therapie der Dystonie in spezialisierten Zentren:

- Botulinumtoxin bei ausgewählten schweren Fällen

- Baclofen intrathecal über eine implantierte Pumpe

\section{Das Wichtigste für die klinische Praxis}

Ein CRPS kann ab circa 6 Wochen nach Extremitätentrauma bei typischen Symptomen an den distalen Gliedmaßen diagnostiziert werden. Die Diagnose wird anhand standardisierter klinischer Kriterien nach sorgfältiger differentialdiagnostischer Abklärung gestellt. Psychologische Risikofaktoren fördern im Krankheitsverlauf Schmerzpersistenz und Funktionsverlust der betroffenen Extremität.

Die akute Entzündung sollte möglichst frühzeitig behandelt werden. Die Schmerztherapie erfolgt mit Analgetika gegen akute und neuropathische Schmerzen. Regelmäßige Physio- und Ergotherapie sind unabdingbare Bestandteile der CRPS-Therapie.

Der Behandlungserfolg hängt wesentlich von der Motivation und der selbstverantwortlichen aktiven Mitbeteiligung des Patienten zur Funktionsverbesserung der betroffenen Extremität ab. 
Interessenkonflikt: Die Autoren erklären, dass keine wirtschaftlichen oder persönlichen Verbindungen bestehen

Danksagung: Die Autoren werden durch Mittel der Berufsgenossenschaft für das Gesundheitswesen (BGW) Mainz unterstützt.

\section{Online zu finden unter}

http://dx.doi.org/10.1055/a-0857-5724

\section{Literatur}

1 Sandroni P, Benrud-Larson LM, McClelland RL et al. Complex regional pain syndrome type I: incidence and prevalence in Olmsted county, a population-based study. Pain 2003; 103 (1-2): 199-207

$\overline{2}$ de Mos M, de Bruijn AG, Dielemann JP et al. The incidence of complex regional pain syndrome: a population-based study. Pain 2007; 129 (1-2): $12-20$

3 Moseley GL, Herbert RD, Parsons T et al. Intense pain soon after wrist fracture strongly predicts who will develop complex regional pain syndrome: prospective cohort study. J Pain 2014; 15 (1): 16-23

$\overline{4}$ Bean DJ, Johnson MH, Heiss-Dunlop W et al. Extent of recovery in the first 12 months of complex regional pain syndrome type-1: A prospective study. Eur J Pain 2016; 20 (6): 884-94

$\overline{5}$ Marinus J, Moseley GL, Birklein F et al. Clinical features and pathophysiology of complex regional pain syndrome. Lancet Neurol 2011; 10 (7): 637-648

$\overline{6}$ Caty G, Hu L, Legrain V et al. Psychophysical and electrophysiological evidence for nociceptive dysfunction in complex regional pain syndrome. Pain 2013; 154 (11): 2521-2528

7 Harden RN, Bruehl S, Perez RS et al. Validation of proposed diagnostic criteria (the "Budapest Criteria") for Complex Regional Pain Syndrome. Pain 2010; 150 (2): 268-274

$\overline{8}$ Birklein F, Drummond PD, Li W et al. Activation of cutaneous immune responses in complex regional pain syndrome. J Pain 2014; 15(5): 485-495
9 Moseley GL, Gallace A, lannetti GD. Spatially defined modulation of skin temperature and hand ownership of both hands in patients with unilateral complex regional pain syndrome. Brain 2012; 135 (12): 3676-3686

$\overline{10}$ Bean DJ, Johnson MH, Heiss-Dunlop W. Do psychological factors influence recovery from complex regional pain syndrome type 1 ? A prospective study. Pain 2015; 156 (11): 2310-2318

$\overline{11}$ Speck V, Schiereth T, Birklein F et al. Increased prevalence of posttraumatic stress disorder in CRPS. Eur J Pain 2017; 21 (3): 466-473

$\overline{12}$ Deutsche Gesellschaft für Neurologie. S1 Leitlinie Diagnostik und Therapie komplexer regionaler Schmerzsymptome (CRPS) (2018). Im Internet: https://www.dgn.org/leitlinien/3618-II030-116-diagnostik-und-therapie-komplexer-regionaler-schmerzsyndrome-crps-2018hen; Stand: 15.02.2019

$\overline{13}$ van de Vusse AC, Stomp-van den Berg SG, Kessels AH et al. Randomised controlled trial of gabapentin in Complex Regional Pain Syndrome type 1 [ISRCTN84121379]. BMC Neurol 2004; 4 (1): 13

$\overline{14}$ Deutsche Gesellschaft für Anästhesiologie und Intensivmedizin, Deutsche Gesellschaft für Angiologie, Deutsche Gesellschaft für Kardiologie et al. S3 Leitlinie Epidurale Rückenmarkstimulation zur Therapie chronischer Schmerzen (2013). Im Internet: http://www.awmf.org/leitlinien/detail/II/041-002.html; Stand: 15.02.2019

$\overline{15}$ Cacchio A, De Blasis E, Necozione S et al. Mirror therapy for chronic complex regional pain syndrome type 1 and stroke. N Engl J Med 2009; 361 (6): 634-636

$\overline{16}$ Moseley GL. Graded motor imagery is effective for long-standing complex regional pain syndrome: a randomised controlled trial. Pain 2004; 108 (1-2): 192-198

$\overline{17}$ den Hollander M, Goossens M, de Jong J et al. Expose or protect? A randomized controlled trial of exposure in vivo vs pain-contingent treatment as usual in patients with complex regional pain syndrome type 1. Pain 2016; 157 (10): 23182329

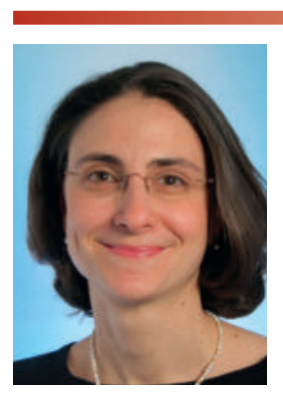

Dr. phil. Dipl.-Psych. Violeta Dimova Langenbeckstraße 1

55131 Mainz

violeta.dimova@unimedizin-mainz.de

Violeta Dimova ist wissenschaftliche Mitarbeiterin der Arbeitsgruppe „Periphere Neurologie und Schmerz" der Klinik und Poliklinik für Neurologie der Universitätsmedizin Mainz. Ihre Forschungsschwerpunkte sind psychologische Risiko- und Resilienzfaktoren, zentrale Schmerzen nach Schlaganfall sowie Neuropsychologie.

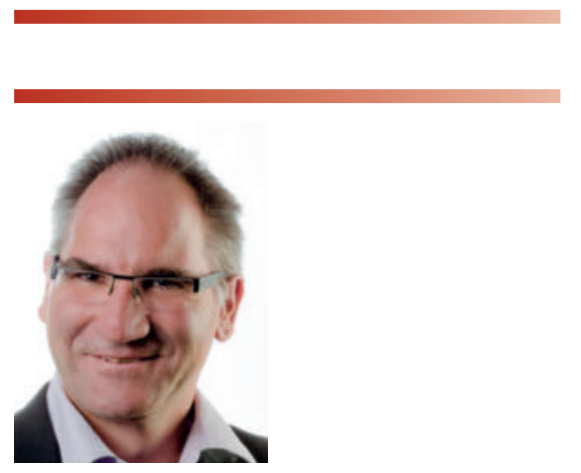

Prof. Dr. med. Frank Birklein Langenbeckstraße 1 55131 Mainz

frank.birklein@unimedizin-mainz.de

Frank Birklein ist Facharzt für Neurologie, Oberarzt und Leiter der Sektion für periphere neurologische Erkrankungen und Schmerz der Klinik und Poliklinik für Neurologie der Universitätsmedizin Mainz. Er ist international ausgewiesener Experte auf dem Gebiet der Schmerzforschung, insbesondere des komplexen regionalen Schmerzsyndroms. 